Article

\title{
Fluctuation-Driven Transport in Biological Nanopores. A 3D Poisson-Nernst-Planck Study
}

\author{
Marcel Aguilella-Arzo, María Queralt-Martín † , María-Lidón Lopez and Antonio Alcaraz * \\ Laboratory of Molecular Biophysics, Department of Physics, Universitat Jaume I, Av. Vicent Sos Baynat s/n, \\ 12071 Castellón, Spain; arzo@uji.es (M.A.-A.); mqueralt@uji.es (M.Q.-M.); lopezp@uji.es (M.-L.L.) \\ * Correspondence: alcaraza@uji.es; Tel.: +34-964-72-8044 \\ + Current affiliation: Program in Physical Biology, Eunice Kennedy Shriver NICHD, National Institutes of \\ Health, Bethesda, MD 20892, USA.
}

Academic Editors: Giancarlo Franzese, Ivan Latella and Miguel Rubi

Received: 28 January 2017; Accepted: 9 March 2017; Published: 14 March 2017

\begin{abstract}
Living systems display a variety of situations in which non-equilibrium fluctuations couple to certain protein functions yielding astonishing results. Here we study the bacterial channel OmpF under conditions similar to those met in vivo, where acidic resistance mechanisms are known to yield oscillations in the electric potential across the cell membrane. We use a three-dimensional structure-based theoretical approach to assess the possibility of obtaining fluctuation-driven transport. Our calculations show that remarkably high voltages would be necessary to observe the actual transport of ions against their concentration gradient. The reasons behind this are the mild selectivity of this bacterial pore and the relatively low efficiencies of the oscillating signals characteristic of membrane cells (random telegraph noise and thermal noise).
\end{abstract}

Keywords: non-equilibrium fluctuations; ion transport; biological channel; electrodiffusion; computational biophysics

\section{Introduction}

Energy-transduction processes occurring in biosystems display astonishing high efficiencies that could be partially explained considering the contribution of non-equilibrium fluctuations [1]. Ratchet mechanisms take advantage of functional anisotropies to bias the response of the system to external fluctuations and produce a net flux of energy [2]. In the case of nanochannels, asymmetric conduction has been shown to be a particularly effective ratchet [3-5]. Thus, when the electric voltage across the membrane fluctuates (due to chemical reactions out of equilibrium or stochastic opening and closing of membrane channels) [6], a net flow of ions (the so-called stochastic pumping or Brownian pumping) could appear without the expenditure of metabolic energy due to the current rectification [3,7].

In a recent study, we showed that OmpF porin, a non-specific channel located in the outer membrane of Escherichia coli (E. coli) [8,9], may perform as a molecular ratchet in conditions designed to mimic acidic stress on bacteria [7]. Using asymmetrically charged lipid bilayers and realistic $\mathrm{pH}$ gradients we demonstrated that zero-average electric potentials similar to those actually measured in E. coli [10] may yield electrical pumping of ions against an external concentration gradient. Interestingly, this uphill transport obtained in the $\mathrm{OmpF}$ channel is directional: depending on the orientation of the concentration gradient, the system can pump either cations or anions from the diluted solution to the concentrated one [7].

The theoretical study of fluctuation-driven transport in $\mathrm{OmpF}$ is complicated by the fact that we deal with a wide mesoscopic channel allowing the simultaneous transport of water molecules and salt cations and anions. Furthermore, at extreme $\mathrm{pH}$ conditions, protons or hydroxyls also contribute themselves to the measured current, changing the permeation pathways of salt ions [11]. Accordingly, 
the relative contribution of each ion to the total current (the channel selectivity) cannot be easily anticipated because of the coupling between electrostatic and diffusional effects arising from the different diffusivities of ions [11]. Here we address this question using the three-dimensional (3D) structure of the channel available at the Protein Data Bank (code 2OMF). We calculate the ion fluxes through the channel using the three-dimensional Poisson-Nernst-Planck (PNP-3D) model and taking into account the salt ions $\left(\mathrm{K}^{+}, \mathrm{Cl}^{-}\right)$and also protons and hydroxyls $\left(\mathrm{H}^{+}, \mathrm{OH}^{-}\right)$.

In principle, the transport of ions against their concentration gradient is attained when the superimposed fluctuating potential is high enough to reverse the direction of the current that is originated by the concentration gradient itself. However, in non-ideally selective channels such as OmpF the reversal of the current could be due not only to the uphill transport of counterions but also to the downhill transport of coions [7,12]. By means of the PNP-3D formalism, we evaluate the individual current carried by each ion under different zero-mean oscillating voltages and discuss the conditions requested to obtain the actual transport of ions against their concentration gradient. We underscore the idea that due to the multiionic character of the channel, the actual uphill transport requires considerably larger potentials than those needed to just reverse the direction of the current.

\section{Materials and Methods}

\subsection{Theoretical Calculations}

The so-called Poisson-Nernst-Planck equations (PNP) are mean-field phenomenological equations that describe ion transport through ionic channels [13-15]. The Poisson equation relates the position-dependent electric charge density to the electrostatic potential of the system, while the contribution from the ions in solution is estimated using the equilibrium Boltzmann equation. Finally, the non-equilibrium ionic fluxes are calculated using the Nernst-Planck equations. The channel fixed charge is obtained by using the University of Houston Brownian Dynamics (UHBD) code $[16,17]$ to calculate the apparent $p K_{a}$ of the channel residues at the particular $\mathrm{pH}$ configuration of our study, using the three-dimensional structure of the OmpF channel (Protein Data Bank code: 2OMF) as obtained from X-ray analysis [18]. A more detailed description of the method employed can be found in [11], including the implementation of $\mathrm{H}^{+}$and $\mathrm{OH}^{-}$ions into the system. The numerical solution of the system equations has been obtained using FiPy [19], a solver of partial differential equations written in Python [20], as described in detail elsewhere [11]. The existence of a charged membrane was simulated adding a small charged region, $5 \AA$ wide, at one of the channel sides, over the ion inaccessible membrane region. The charge was adjusted to mimic a surface charge of about 1 negative elementary charge every $44.2 \AA^{2}$ (approximately the area per lipid for dipalmitoyl phosphatidylcholine (DPPS) lipid [21]).

\subsection{Experimental Methods}

Wild-type OmpF, kindly provided by Dr. S. M. Bezrukov (NICHD, NIH, Bethesda, MD, USA), was isolated and purified from an E. coli culture. Planar membranes were formed by the apposition of monolayers across orifices with diameters of 70-100 $\mu \mathrm{m}$ on a 15- $\mu \mathrm{m}$-thick Teflon partition using diphytanoyl phosphatidylcholine (DPhPC) or diphytanoyl phosphatidylserine (DPhPS). The orifices were pre-treated with a $1 \%$ solution of hexadecane in pentane. An electric potential was applied using $\mathrm{Ag} / \mathrm{AgCl}$ electrodes in $2 \mathrm{M} \mathrm{KCl}, 1.5 \%$ agarose bridges assembled within standard $250 \mathrm{~mL}$ pipette tips. The potential was defined as positive when it was higher on the side of the protein addition (the cis side of the membrane chamber), whereas the trans side was set to ground. An Axopatch 200B amplifier (Molecular Devices, Sunnyvale, CA, USA) in the voltage-clamp mode was used to measure the current and applied potential. The signal was digitalized at $50 \mathrm{kHz}$ sampling frequency after $10 \mathrm{kHz} 8$-pole in-line Bessel filtering. The chamber and the head stage were isolated from external noise sources with a double metal screen (Amuneal Manufacturing Corp., Philadelphia, PA, USA). 
The $\mathrm{pH}$ was adjusted by adding $\mathrm{HCl}$ or $\mathrm{KOH}$ and controlled during the experiments with a GLP22 $\mathrm{pH}$ meter (Crison, Barcelona, Spain). Measurements were obtained at $\mathrm{T}=(23 \pm 1.5){ }^{\circ} \mathrm{C}$.

\section{Results and Discussion}

\subsection{The Importance of Membrane Charge Asymmetry}

The mechanisms by which $E$. coli can survive inside the stomach under considerable acidic stress ( $\mathrm{pH} 2 \sim 3)$ are still under debate [22-26]. In particular, the role of the outer membrane and its constituents remains mostly unexplored. The existing literature focuses on how proton influx is reduced by changes in membrane fluidity [10], albeit the possible changes in the membrane charge are not examined in detail. Recent experimental studies mentioned before highlight the crucial role of lipid charges on $\mathrm{OmpF}$ channel conductance [7], providing the current rectification required for the ratchet mechanism. We tackle this issue by considering that because of the acidic stress, the outer membrane could become asymmetrically charged. We represent this situation by using negatively charged lipids in the inner side kept at neutral $\mathrm{pH}$ and neutral lipids in the outer monolayer facing an acidic solution (representing the titration of the lipid polar heads at low $\mathrm{pH}$ [27]). Although this is a convenient minimal model to explore the role of electrostatic interactions, we are aware that this is a major simplification. In fact, the outer membrane of $E$. coli is a heterogeneous mixture of lipopolysaccharides (LPS) and phospholipids [28] that differs significantly from plasma membranes [29]. The outer leaflet is almost exclusively comprised of LPS, which are a very effective barrier for the spontaneous diffusion of lipophilic compounds due to the low fluidity of the LPS hydrocarbon domain and the strong lateral interactions between LPS molecules [28]. The inner leaflet is a mixture of cardiolipin and phospholipids (PG and PE) so that charged lipids are in a similar proportion to that found in endoplasmatic reticulum membranes [28,29].

Figure 1 shows that asymmetric conduction can be achieved in symmetrical neutral membranes (PC I I PC, Figure 1a) and asymmetrically charged membranes (PC I PS, Figure 1b) when there is a realistic $\mathrm{pH}$ gradient across the channel. The PNP-3D model calculations show a good agreement not only with the experimental recordings shown in the inset of each figure but also with previous experimental studies [12,30]. In neutral membranes (Figure 1a), both the total current and the current carried by the prevailing counterions $\left(\mathrm{Cl}^{-}\right.$ions in this case) are asymmetric. Figure $1 \mathrm{~b}$ shows the importance of membrane charges. In asymmetrical membranes, the current rectification is slightly higher, but the major change is that now the current is dominated by cations, in contrast to the results shown in Figure 1a. This change in the channel selectivity can be crucial for the acid resistance mechanisms of the bacteria, requiring a precise control of positively charged protons [10].
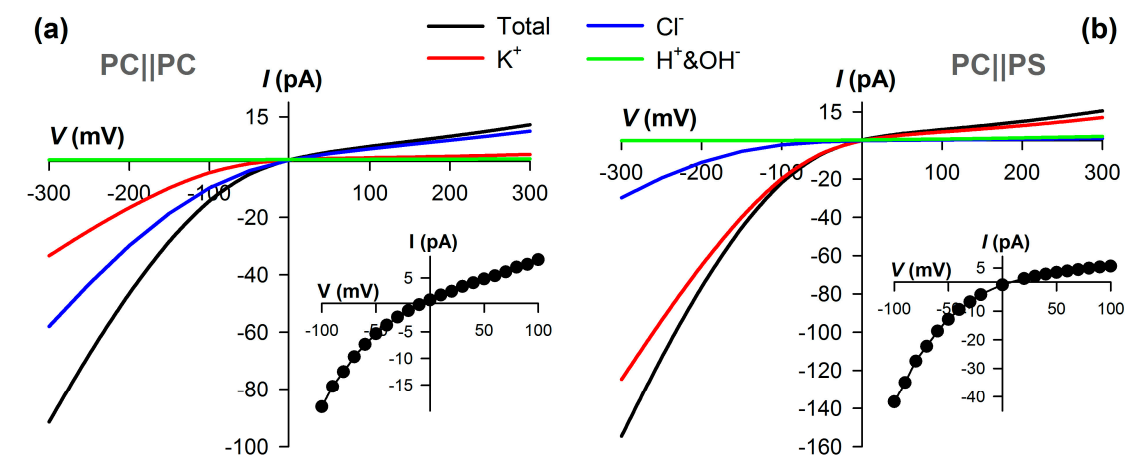

Figure 1. (a) Calculated current-voltage $(I-V)$ curve of OmpF channel in the 3|| 7 configuration for symmetrical neutral membranes PC। I PC; (b) Calculated current-voltage $(I-V)$ curve of OmpF channel in the $\mathrm{pH} 3$ | | 7 configuration for asymmetrical membranes PC | | PS. KCl concentration is $25 \mid$ | $25 \mathrm{mM}$. The insets show a representative experimental $I-V$ curve of OmpF single channel at the same conditions as that of the main figure. 
The calculations provide new insights about the structural origin of current rectification. Figure 2a compares the electrostatic potential across the OmpF channel for uncharged (left) and asymmetrically charged membrane (right). When the membrane is neutral, the titration of acidic residues in the channel mouth facing the acidic solution creates an inhomogeneous charge distribution yielding an overall positive potential, that is, the channel is anion-selective as shown in Figure 1a. Contrariwise, when negative charges are placed in the membrane facing the neutral solution, the balance changes and now the electrostatic potential in the system is more negative, meaning that the channel prefers positive ions as depicted in Figure 1b. Of note, the membrane charge not only regulates the electrostatic potential in the corresponding channel mouth, but it affects the whole system. From the PNP system of equations we also obtained three-dimensional diagrams displaying the trajectories of ions through the channel. Figure $2 \mathrm{~b}$ displays an example of this complete $3 \mathrm{D}$ view for a monomer, at the same conditions as in Figure $1 \mathrm{~b}(\mathrm{KCl} 25$ | I $25 \mathrm{mM}$, neutral I I negative membrane, $\mathrm{pH} 3 \mid$ | 7$)$ and no applied voltage. Remarkably, protons flow down the $\mathrm{pH}$ gradient, inducing the flow of salt ions $\left(\mathrm{Cl}^{-}\right.$and $\mathrm{H}^{+}$from left to right and $\mathrm{K}^{+}$from right to left) in order to fulfill electroneutrality requirements. The importance of membrane charges is graphically demonstrated by the way in which the cation streamlines bend at the pore exit trying to approach to the negatively charged lipid heads. Figure $2 \mathrm{~b}$ demonstrates as well that $\mathrm{K}^{+}$and $\mathrm{Cl}^{-}$ions follow well-separated screw-like trajectories when crossing the channel [31], a characteristic mechanism of some bacterial porins that is not lost despite the large $\mathrm{pH}$ gradients used in our study [11]. This peculiarity is also suggested in the electrostatic maps around the central constriction of the channel in Figure 2a.

(a)

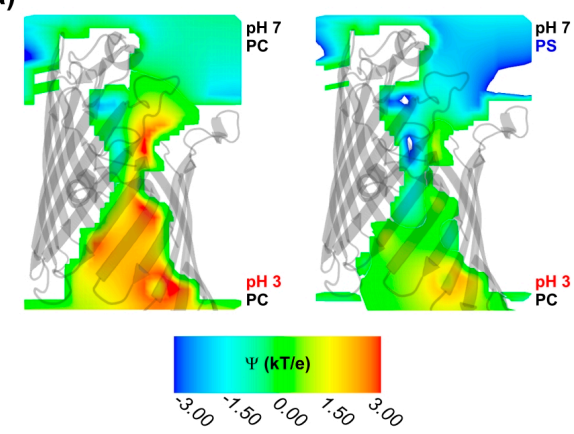

(b)

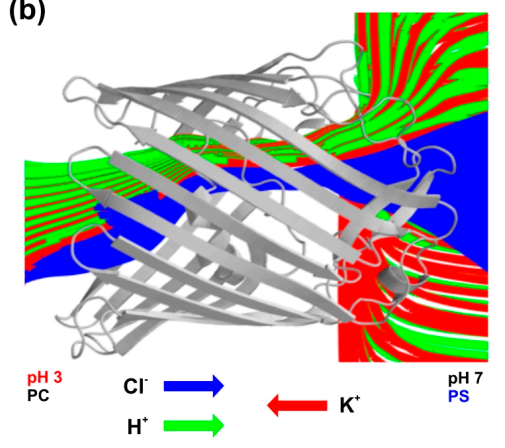

Figure 2. (a) Electrostatic potential in $\mathrm{kT} / \mathrm{e}$ units across OmpF for an uncharged membrane (PC I I PC, left) and an asymmetrically charged membrane (PCI I PS, right); (b) 3D ion current paths along the OmpF channel (in grey) for an asymmetrically charged membrane (PC I IPS). Ions are represented as streamlines to show the paths followed on average by each ion, not taking into account the flux values. Figures were obtained from PNP-3D calculations and represented using Mayavi, a Python-based program for 3D representation. The conditions used were $\mathrm{KCl} 25$ | | $25 \mathrm{mM}$, pH 3 | | 7, and no applied electrostatic potential through the system.

\subsection{Structural Basis of Ion Pumping}

The distinctive feature of large $\beta$-barrel channels such as OmpF with respect to Na-channels, $\mathrm{K}$ channels or $\mathrm{Cl}$ channels is that they do not transport a unique chemical species, but allow the simultaneous transport of all of them present in the solution [32]. Hence, when a salt concentration gradient is imposed, both anions and cations flow from the concentrated to the diluted side of the membrane but contribute differently to the overall current as imposed by the channel selectivity. Figure 3 a shows structure-based calculations of the $I-V$ curve considering a salt gradient $c_{c i s}>c_{\text {trans }}$ and the asymmetrically charged membrane. We can observe that the channel is selective to cations, in excellent agreement with the electrophysiological recordings, which are shown in the inset of the figure [7]. Notably, the existence of a concentration gradient restrains the ability of the channel to rectify the current, but the $I-V$ curve still displays a significant asymmetry for the total and $\mathrm{K}^{+}$current. 
Figure $3 \mathrm{~b}$ shows the concentration profile of $\mathrm{K}^{+}$and $\mathrm{Cl}^{-}$ions in the $\mathrm{pH}$ and concentration conditions of Figure 3a, but at two different polarities: +200 and $-200 \mathrm{mV}$. Positive voltages promote the downhill transport of $\mathrm{K}^{+}$while hindering the transport of $\mathrm{Cl}^{-}$ions that could hardly reach the diluted side. In contrast, negative voltages permit the downhill transport of $\mathrm{Cl}^{-}$, but at the same time provide a dominant current of $\mathrm{K}^{+}$that does not differ much from that found at positive voltages.
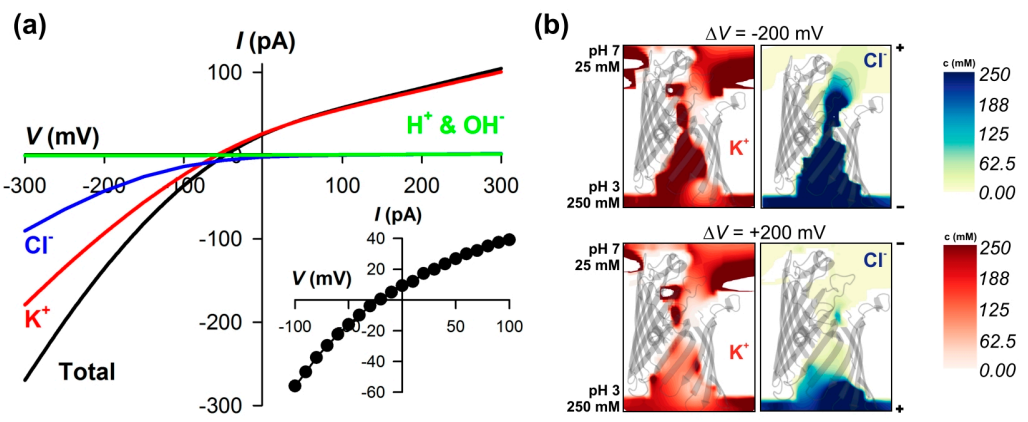

Figure 3. (a) Calculated current-voltage $(I-V)$ curve of OmpF channel in the 3|| 7 configuration for concentration ratio $\mathrm{r}=10(\mathrm{KCl} 250|| 25 \mathrm{mM})$ and asymmetrical membranes PC I | PS. The inset shows an experimental $I-V$ curve at the same conditions as in the main figure; (b) Concentration profiles for potassium and chloride ions across the OmpF channel (in gray) under an applied potential of $+200 \mathrm{mV}$ and $-200 \mathrm{mV}$, as indicated. The conditions used for the calculations are the same as in (a).

To evaluate if the asymmetry observed in Figure 3 may lead to an actual pumping of counterions (cations in this case), we used different types of zero-mean fluctuation potentials. The oscillating voltage signals that appear in living cells may come from dissipative processes yielding thermal noise [2] but could also have other origins, such as the stochastic opening and closing of membrane channels in the membrane or the occurrence of non-equilibrium chemical reactions. Typically, these later signals are simulated using random telegraph noise (RTN) with different voltage distributions [33,34]. In particular, we used voltages fluctuating between two extreme values and also following a Gaussian and a random distribution. Figure 4 (left panel) shows these input signals and the outputs obtained, based on the $I-V$ curve of Figure $3 a$.

Numerically solving the PNP system of equations allows us to obtain separately the current output of each ion, as shown in the left panels of Figure 4. In all cases, we consider that the output signal is the slave of the input one, as shown in the corresponding experimental situations [7]. As could be expected from the cationic selectivity displayed in Figure 3, the total current and the current carried by cations are pretty similar, although $\mathrm{I}_{\text {total }}$ is always larger at negative values thanks to the contribution of anions. This is clearly seen in the right panel of Figure 4, where the voltage and current distributions are displayed. The distributions of current are biased, reflecting the asymmetry of the output signals, which may imply an eventual pumping of cations.

The qualitative message of Figure 4 is appealing but a quantitative analysis is desirable. A more convenient way of probing the pumping of ions is calculating the average currents for both the total current and the current carried by each ion, as shown in Figure 5 for each signal.

The average total current turns from positive at low potentials to negative at high potentials, for all signals displayed. As mentioned before, this means that the superimposed fluctuating potential is high enough to outweigh the current originated by the concentration gradient. However, due to the multiionic character of the channel, one may wonder whether the change in the average current is enough to assure the electrical pumping of ions [12]. In the case of $\mathrm{Cl}^{-}$ions, their average current is always negative, which indicates that these ions always flow downhill (i.e., from the concentrated to the diluted solution) regardless the magnitude of the applied voltage. However, the behavior of $\mathrm{K}^{+}$ions is completely different: they display a positive average current $\left(<I_{K}>>0\right)$ at low potentials that turns into a negative current when the applied voltage is high enough. That is, they present an uphill transport 
at sufficiently high potentials. Interestingly, the voltage at which $\left\langle I_{K}\right\rangle<0$ depends on the type of signal, indicating that some signals are more efficient than others. Among those analyzed here, the most efficient is that operating at constant $\mathrm{V}$. This could be the signal found when voltage oscillations come from the flickering of a unique type of membrane pore operating between well-defined current states [6]. For these conditions, the actual pumping would occur at moderate voltage values around $100-150 \mathrm{mV}$. Such a voltage configuration may not be the most probable one, having in mind that in the cell membrane there is actually a wide variety of diverse membrane channels that furthermore usually display several subconductance states [9]. Voltage configurations depicting more realistic fluctuations (Gaussian or randomly distributed ones) require considerably higher voltages to obtain $<I_{\mathrm{K}}><0$. A potential larger than $250 \mathrm{mV}$ seems unlikely to appear in the bacterial membrane (voltage fluctuations are typically between $-100 \mathrm{mV}$ and $+100 \mathrm{mV}$ [10]), but we do not aim to establish a quantitative connection between the situation in vivo and the mechanism depicted here. We are aware that in vitro experiments and in silico calculations shown here provide only a qualitative picture that, although appealing, could differ from the actual one. On the other side, the conclusions of Figure 5 are not limited to RTN, but they can be extended to white noise or thermal noise. For the sake of simplicity, here we use a triangular-shaped signal (see Figure 6) which gives essentially the same average as any random signal. Again, the distributed voltage means a lower efficiency of the pumping mechanism so that voltages around $300 \mathrm{mV}$ are required to obtain a transport of $\mathrm{K}^{+}$against their concentration gradient.
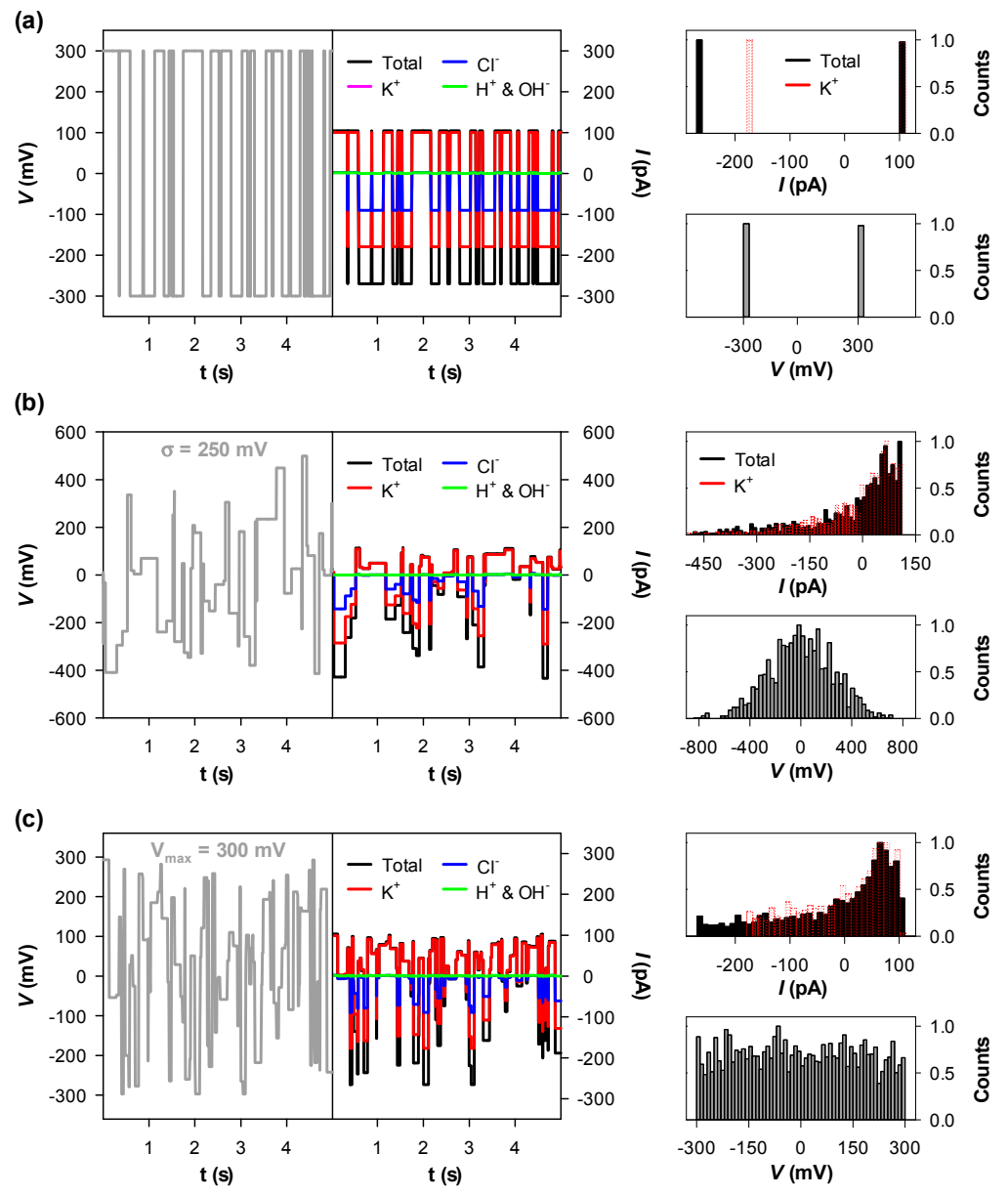

Figure 4. Left panel: Representative calculated traces showing different input signals (constant voltage (a); Gaussian-distributed voltage (b); and random noise (c) and the corresponding output currents; Right panel: Voltage and current (total and $\mathrm{K}^{+}$current) distributions obtained from the signals as those shown in the left panel. 

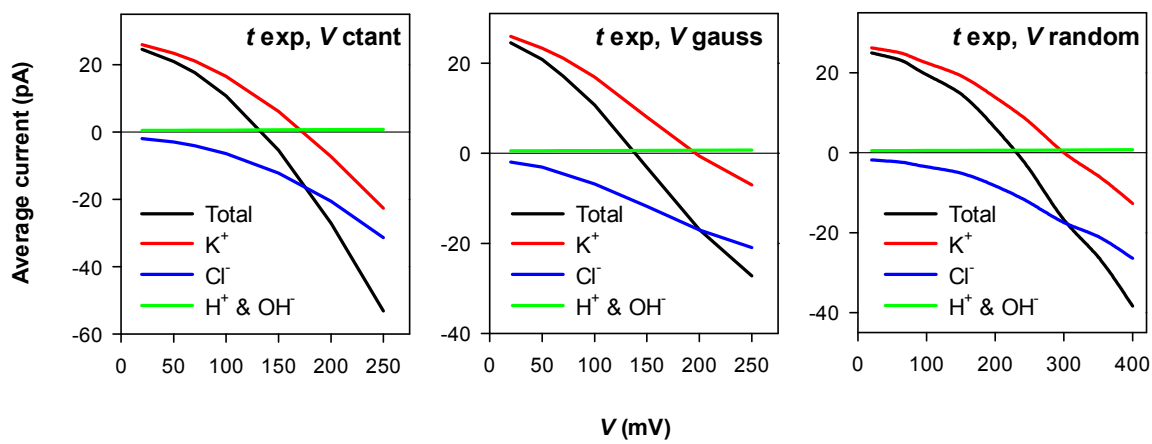

Figure 5. Average current as a function of voltage, calculated from the signals showed in Figure 4.

(a)

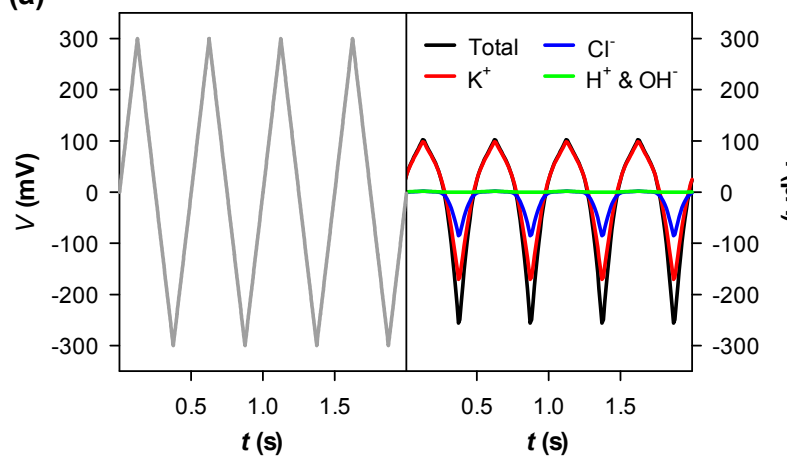

(b)

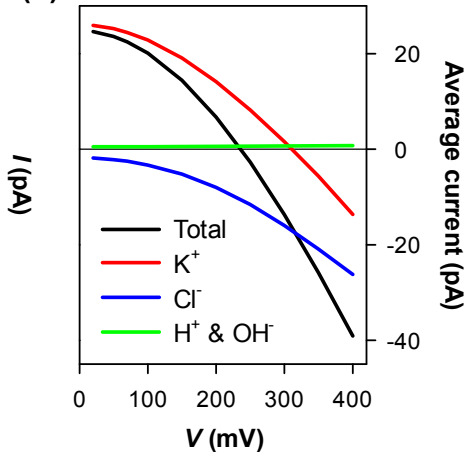

Figure 6. (a) Calculated traces showing a triangular input voltage and the corresponding output current. The $\mathrm{V}$ and I distributions are almost identical to that obtained for a random distribution (right panel of Figure 4b); (b) Calculated average current as a function of voltage, from the signal in the left panel.

In summary, our calculations show that the voltage requested to obtain the uphill transport of counterions is significantly increased by the poor selectivity of the pore allowing the downhill transport of coions. Furthermore, the oscillating signals characteristic of membrane cells (RTN and thermal noise) display relatively low efficiencies so that remarkably high voltages, at the limit of what could be realistic, would be necessary to observe actual electrical pumping of ions from the diluted side to a more concentrated one. Our analysis is limited to certain types of signals but there could be other processes contributing to the channel dynamics that facilitate the pumping process. It has been shown that the permeation of ions could be enhanced by transient fluctuations in the channel shape. Such so-called "breathing" motions may play a key role in channel gating as voltage sensors [35-37].

Acknowledgments: Authors acknowledge the financial support by the Ministry of Economy and Competitiveness of Spain (Project No. FIS2013-40473-P and FIS2016-75257-P), and Universitat Jaume I (Project No. P1.1B2015-28).

Author Contributions: A.A. and M.Q.-M. conceived and designed the calculations; M.A.-A. performed the numerical calculations; M.-L.L. performed the experiments; A.A. and M.Q.-M. analyzed the data and wrote the manuscript.

Conflicts of Interest: The authors declare no conflict of interest.

\section{References}

1. Westerhoff, H.V.; Tsong, T.Y.; Chock, P.B.; Chen, Y.D.; Astumian, R.D. How enzymes can capture and transmit free energy from an oscillating electric field. Proc. Natl. Acad. Sci. USA 1986, 83, 4734-4738. [CrossRef] [PubMed]

2. Astumian, R.D. Thermodynamics and kinetics of a Brownian motor. Science 1997, 276, 917-922. [CrossRef] [PubMed] 
3. Verdiá-Báguena, C.; Queralt-Martín, M.; Aguilella, V.M.; Alcaraz, A. Protein ion channels as molecular ratchets. Switchable current modulation in Outer Membrane Protein F porin induced by millimolar $\mathrm{La}^{3+}$ ions. J. Phys. Chem. C 2012, 116, 6537-6542. [CrossRef]

4. Ramirez, P.; Ali, M.; Ensinger, W.; Mafe, S. Information processing with a single multifunctional nanofluidic diode. Appl. Phys. Lett. 2012, 101, 133108. [CrossRef]

5. Ramirez, P.; Gomez, V.; Ali, M.; Ensinger, W.; Mafe, S. Net currents obtained from zero-average potentials in single amphoteric nanopores. Electrochem. Commun. 2013, 31, 137-140. [CrossRef]

6. Mosgaard, L.D.; Zecchi, K.A.; Heimburg, T.; Budvytyte, R. The effect of the nonlinearity of the response of lipid membranes to voltage perturbations on the interpretation of their electrical properties. A new theoretical description. Membranes 2015, 5, 495-512. [CrossRef] [PubMed]

7. López, M.L.; Queralt-Martín, M.; Alcaraz, A. Stochastic pumping of ions based on colored noise in bacterial channels under acidic stress. Nanoscale 2016, 8, 13422-13428. [CrossRef] [PubMed]

8. Delcour, A.H. Solute uptake through general porins. Front. Biosci. 2003, 8, D1055-D1071. [CrossRef] [PubMed]

9. Nikaido, H. Molecular Basis of Bacterial Outer Membrane Permeability Revisited. Microbiol. Mol. Biol. Rev. 2003, 67, 593-656. [CrossRef] [PubMed]

10. Foster, J.W. Escherichia coli acid resistance: Tales of an amateur acidophile. Nat. Rev. Microbiol. 2004, 2, 898-907. [CrossRef] [PubMed]

11. Queralt-Martín, M.; Peiró-González, C.; Aguilella-Arzo, M.; Alcaraz, A. Effects of extreme pH on ionic transport through protein nanopores: The role of ion diffusion and charge exclusion. Phys. Chem. Chem. Phys. 2016, 18, 21668-21675. [CrossRef] [PubMed]

12. Queralt-Martín, M.; García-Giménez, E.; Aguilella, V.M.; Ramirez, P.; Mafe, S.; Alcaraz, A. Electrical pumping of potassium ions against an external concentration gradient in a biological ion channel. Appl. Phys. Lett. 2013, 103, 43707. [CrossRef]

13. Lakshminarayanaiah, N. Equations of Membrane Biophysics; Academic Press: New York, NY, USA, 1984.

14. Nonner, W.; Eisenberg, B. Ion permeation and glutamate residues linked by Poisson-Nernst-Planck theory in L-type calcium channels. Biophys. J. 1998, 75, 1287-1305. [CrossRef]

15. Kurnikova, M.G.; Coalson, R.D.; Graf, P.; Nitzan, A. A Lattice Relaxation Algorithm for Three-Dimensional Poisson-Nernst-Planck Theory with Application to Ion Transport through the Gramicidin A Channel. Biophys. J. 1999, 76, 642-656. [CrossRef]

16. Davis, M.E.; Madura, J.D.; Luty, B.A.; McCammon, J.A. Electrostatics and diffusion of molecules in solution: Simulations with the University of Houston Brownian Dynamics program. Comput. Phys. Commun. 1991, 62, 187-197. [CrossRef]

17. Madura, J.D.; Briggs, J.M.; Wade, R.C.; Davis, M.E.; Luty, B.A.; Ilin, A.; Antosiewicz, J.; Gilson, M.K.; Bagheri, B.; Scott, L.R.; et al. Electrostatics and diffusion of molecules in solution: Simulations with the University of Houston Brownian Dynamics program. Comput. Phys. Commun. 1995, 91, 57-95. [CrossRef]

18. Cowan, S.; Garavito, R.; Jansonius, J.; Jenkins, J.; Karlsson, R.; König, N.; Pai, E.; Pauptit, R.; Rizkallah, P.; Rosenbusch, J.; et al. The structure of OmpF porin in a tetragonal crystal form. Structure 1995, 3, 1041-1050. [CrossRef]

19. Guyer, J.E.; Wheeler, D.; Warren, J.A. FiPy: Partial Differential Equations with Python. Comput. Sci. Eng. 2009, 11, 6-15. [CrossRef]

20. Van Rossum, G.; Drake, F.L. PYTHON 2. 6 Reference Manual: (Python Documentation MANUAL Part 2); Python Software Foundation: Beaverton, OR, USA, 2009.

21. Marr, J.M.; Li, F.; Petlick, A.R.; Schafer, R.; Hwang, C.-T.; Chabot, A.; Ruggiero, S.T.; Tanner, C.E.; Schultz, Z.D. The Role of Lateral Tension in Calcium-Induced DPPS Vesicle Rupture. Langmuir 2012, 28, 11874-11880. [CrossRef] [PubMed]

22. Gorden, J.; Small, P.L. Acid resistance in enteric bacteria. Infect. Immun. 1993, 61, 364-367. [PubMed]

23. Castanie-Cornet, M.P.; Penfound, T.A.; Smith, D.; Elliott, J.F.; Foster, J.W. Control of acid resistance in Escherichia coli. J. Bacteriol. 1999, 181, 3525-3535. [PubMed]

24. Lin, J.; Lee, I.S.; Frey, J.; Slonczewski, J.L.; Foster, J.W. Comparative analysis of extreme acid survival in Salmonella typhimurium, Shigella flexneri, and Escherichia coli. J. Bacteriol. 1995, 177, 4097-4104. [CrossRef] [PubMed] 
25. Diez-Gonzalez, F.; Russell, J.B. The ability of Escherichia coli O157:H7 to decrease its intracellular $\mathrm{pH}$ and resist the toxicity of acetic acid. Microbiology 1997, 143 Pt 4, 1175-1180. [CrossRef] [PubMed]

26. Jordan, K.N.; Oxford, L.; O’Byrne, C.P. Survival of low-pH stress by Escherichia coli O157:H7: Correlation between alterations in the cell envelope and increased acid tolerance. Appl. Environ. Microbiol. 1999, 65, 3048-3055. [PubMed]

27. Tyäuble, H.; Teubner, M.; Woolley, P.; Eibl, H. Electrostatic interactions at charged lipid membranes. I. Effects of $\mathrm{pH}$ and univalent cations on membrane structure. Biophys. Chem. 1976, 4, 319-342. [CrossRef]

28. Nikaido, H.; Vaara, M. Molecular basis of bacterial outer membrane permeability. Microbiol. Rev. 1985, 49, 1-32. [CrossRef] [PubMed]

29. van Meer, G.; Voelker, D.R.; Feigenson, G.W. Membrane lipids: Where they are and how they behave. Nat. Rev. Mol. Cell Biol. 2008, 9, 112-124. [CrossRef] [PubMed]

30. Alcaraz, A.; Ramírez, P.; García-Giménez, E.; López, M.L.; Andrio, A.; Aguilella, V.M. A pH-tunable nanofluidic diode: Electrochemical rectification in a reconstituted single ion channel. J. Phys. Chem. B 2006, 110, 21205-21209. [CrossRef] [PubMed]

31. Im, W.; Roux, B. Ions and counterions in a biological channel: A molecular dynamics simulation of OmpF porin from Escherichia coli in an explicit membrane with $1 \mathrm{M} \mathrm{KCl}$ aqueous salt solution. J. Mol. Biol. 2002, 319, 1177-1197. [CrossRef]

32. Aguilella, V.M.; Queralt-Martín, M.; Aguilella-Arzo, M.; Alcaraz, A. Insights on the permeability of wide protein channels: Measurement and interpretation of ion selectivity. Integr. Biol. 2011, 3, 159-172. [CrossRef] [PubMed]

33. Fuliński, A.; Grzywna, Z.; Mellor, I.; Siwy, Z.; Usherwood, P. Non-Markovian character of ionic current fluctuations in membrane channels. Phys. Rev. E 1998, 58, 919-924. [CrossRef]

34. Xie, T.D.; Marszalek, P.; Chen, Y.D.; Tsong, T.Y. Recognition and processing of randomly fluctuating electric signals by Na,K-ATPase. Biophys. J. 1994, 67, 1247-1251. [CrossRef]

35. Danelon, C.; Nestorovich, E.M.; Winterhalter, M.; Ceccarelli, M.; Bezrukov, S.M. Interaction of zwitterionic penicillins with the OmpF channel facilitates their translocation. Biophys. J. 2006, 90, 1617-1627. [CrossRef] [PubMed]

36. Rui, H.; Il Lee, K.; Pastor, R.W.; Im, W. Molecular dynamics studies of ion permeation in VDAC. Biophys. J. 2011, 100, 602-610. [CrossRef] [PubMed]

37. Shrivastava, I.H.; Sansom, M.S. Simulations of ion permeation through a potassium channel: Molecular dynamics of KcsA in a phospholipid bilayer. Biophys. J. 2000, 78, 557-570. [CrossRef] 